\section{Co-creación de valor en pequeños agronegocios a través de redes sociales}

\author{
Co-creation of value in small agribusinesses \\ through social networks
}

\begin{abstract}
RESUMEN
La presente investigación buscó conocer el potencial de co-creación de valor de los agronegocios mediante las redes sociales de los pequeños productores rurales. Para ello se realizó un estudio de enfoque cualitativo, mediante entrevistas a 14 participantes que forman parte del Programa AGRO RURAL y otros especialistas en marketing digital y redes sociales. Los hallazgos permitieron dividir la información en cuatro categorías para considerar la co-creación de los productos agrarios: a) Potencial del productor, b) Motivación del productor c) Factibilidad de la co-creación mediante las redes sociales y d) Condiciones necesarias. Se concluyó que los pequeños productores están dispuestos a realizar acciones de co-creación, sin embargo, tienen limitaciones en cuanto a conocimiento y habilidades para el uso de la tecnología. Además, la co-creación de los productos agrarios a través de las redes sociales sería de mucho beneficio para los productores para lograr construir y desarrollar marcas de la mano de los consumidores.
\end{abstract}

Palabras clave: Agricultura familiar; productos agrarios; innovación.

\section{ABSTRACT}

This research sought to know the potential for co-creation of value of agribusiness through the social networks of small rural producers. For this, a qualitative approach study was carried out, through interviews with 21 participants who are part of the AGRO RURAL Program and other specialists in digital marketing. The findings allowed us to divide the information into four categories to consider the co-creation of agricultural products: a) Potential of the producer, b) Motivation of the producer c) Feasibility of co-creation through social networks and d) Necessary conditions. It was concluded that small producers are willing to carry out co-creation actions, however, they have limitations in terms of knowledge and skills for the use of technology. In addition, the co-creation of agricultural products through social networks would be of great benefit to producers in order to build and develop brands with consumers.

Keywords: Family farming; agricultural products; innovation.

\section{Elizabeth Emperatriz García Salirrosas ${ }^{1}$} egarcias@untels.edu.pe

\section{Isabel Zurich Geñua} Lizunde $^{1}$

izaz.11.23@hotmail.com

\section{Marisol Victoria Pariona}

Huamaní ${ }^{1}$

mparionahuamani@gmail.com

\footnotetext{
${ }^{1}$ Universidad Nacional Tecnológica de Lima Sur
}

Presentado: 16/09/2020 - Aceptado: 12/10/2020 - Publicado: 26/11/2020

(c) Los autores. Este artículo es publicado por Gestión en el Tercer Milenio de la Facultad de Ciencias Administrativas de la Universidad Nacional Mayor de San Marcos. Este es un artículo de acceso abierto, distribuido bajo los términos de la licencia Creative Commons Atribucion - No Comercia_Compartir Igual 4.0 Internacional. (http://creativecommons.org/licenses/by-nc-sa/4.0/) que permite el uso no comercial, distribución y reproducción en cualquier medio, siempre que la obra original sea debidamente citada. 


\section{INTRODUCCIÓN}

Las Naciones Unidas desde los años 90 están preocupados por combatir la pobreza, el hambre, la desigualdad y abordar el cambio climático. Es así que en el año 2015 presentaron los Objetivos de Desarrollo Sostenible (ODS) para ser alcanzados para el año 2030 (Naciones Unidas, 2018). Para lograr estos objetivos, el desarrollo de la agricultura tiene un papel estratégico en la agenda, debido a que su crecimiento incide directamente en la reducción de la pobreza y la erradicación del hambre, siendo la agricultura familiar la que más contribuye a la creación de trabajo, la producción agrícola sostenible y al crecimiento económico conservando la biodiversidad, aportando a la seguridad alimentaria y a la nutrición (Instituto Interamericano de Cooperación para la Agricultura -IICA, 2010).

La Comisión Económica para América Latina y el Caribe (CEPAL), La Organización de las Naciones Unidas para la Alimentación y la Agricultura (FAO) y el Instituto Interamericano de Cooperación para la Agricultura (IICA) señalan que la agricultura familiar significa más del 50\% del empleo en el sector agropecuario en 14 países de la región y que el cierre de las brechas salariales y el aumento de la productividad podrían contribuir a fortalecer la sostenibilidad económica y social (CEPAL, FAO ,IICA, 2019), lo cual se relaciona con el objetivo número ocho de la agenda al 2030 que consiste en lograr el trabajo decente y el crecimiento económico. Lo cual no será posible para los pequeños agricultores si no se logra un incremento de sus ingresos, puesto que aún están en pobreza.

Los pequeños productores cubren el 70\% de las necesidades alimentarias mundiales y los agronegocios son fuente importante de empleo y de ingresos en todo el mundo. Mejorar la sostenibilidad de las cadenas de valor alimentarias puede beneficiar a millones de hogares pobres en países en desarrollo, así como garantizar el acceso alimentario nutritivo para todos (FAO, 2018).

En el Perú, el sector agrícola en los últimos 20 años ha sido la segunda actividad con mayor rentabilidad después de la minería, siendo así uno de los sectores más importantes y productivos para la exportación de productos no tradicionales. Ha sido considerado el primer productor de espárragos, quinua y maca; seguidos por palta y alcachofas (Castro, Goicochea, \& Flores, 2018). El sector agroexportador se caracteriza por estar enfocado en frutas y hortalizas no tradicionales, cultivados en varios departamentos (Flores Calle, 2014). El sector representa el 8\% del PBI y emplea alrededor del 26\% de la Población Económicamente Activa (PEA).

Con la finalidad de mejorar la calidad de vida de las familias que dependen de la agricultura familiar y reducir la pobreza del sector rural el caso peruano existen leyes y protocolos, como: la Ley de Promoción y Desarrollo de la Agricultura Familiar (Ley N ${ }^{\circ} 30355$, 04.11.2015) y su reglamento (Reglamento $\mathrm{N}^{\circ}$ 015-2016-MINAGRI, 23.07.2016), así mismo se tiene la Estrategia Nacional de Agricultura Familiar 2015-2021 (D.S. $N^{\circ} 009-2015-M I N A G R I$, 20.06.2015) y el Plan Nacional de Agricultura Familiar 2016-2021(en proceso). Reconociendo de esta manera su importancia y rol en la dinamización de las economías locales y en la contribución al empleo rural (Convención Nacional del Agro Peruano [CONVEAGRO], 2018).

El 13 de marzo del 2008 el gobierno peruano por medio del Decreto Legislativo $N^{\circ} 997$ promulgó la creación del Programa de Desarrollo Productivo Agrario Rural - AGRO RURAL, con el fin de promover el desarrollo de la agricultura rural, a través de la provisión de fondos de inversión pública en zonas rurales en el ámbito agrario en territorios menos desarrollados económicamente (Congreso de la República, 2008). Una de las actividades de este programa es insertar al pequeño productor al mercado, puesto que ese es una de las grandes dificultades que presenta este sector.

El término co-creación consiste en hacer partícipe a los consumidores en el proceso del desarrollo de productos o servicios, por lo tanto, pueden contribuir al diseño, el precio y la promoción de los productos de la empresa y de esa forma generar valor (Bettiga, Lamberti, \& Noci, 2018). La co-creación es una de las diversas estrategias que existen en la actualidad para lograr el crecimiento y desarrollo de los negocios, la cual está relacionada con la satisfacción del consumidor (Jiménez-Barreto \& Campo-Martínez, 2018). 
Aunque el término co-creación no es nada nuevo, ahora ha recibido más atención por parte de académicos y profesionales, principalmente debido al uso creciente de las herramientas de Internet y las redes sociales, que permiten una mayor interacción entre consumidores y empresas, a medida que las empresas se esfuerzan por ser diferentes de sus competidores (Martini, Massa, \& Testa, 2012). La colaboración conjunta puede aumentar la eficiencia e incrementar las ganancias a largo plazo (Handayati, Simatupang, \& Perdana, 2015).

La experiencia de co-creación no implica que las empresas intenten complacer a sus clientes, sino que ambos unen esfuerzos para co-construir la experiencia con diálogo activo y experiencia personalizada (Loureiro, Romero, \& Bilro, 2019; Prahalad \& Ramaswamy, 2004) realizan un análisis de co-creación de valor a través del modelo DART con sus cuatro componentes: Diálogo, Acceso, Riesgo / Beneficio y Transparencia.

La combinación de los componentes del modelo DART facilitan que el cliente se integre con la organización, junto con sus experiencias y relaciones; en el contexto de las interacciones, también permite el compromiso entre los involucrados en una red, a fin de promover la co-creación de valor (Donato, Farina, Donaire, \& Dos Santos, 2017).El diálogo se convierte en un flujo multidireccional que facilita la interacción entre los involucrados con un aprendizaje mutuo que genere creatividad e innovación. El acceso se refiere a la disponibilidad, el alcance al conocimiento y la información. El riesgo/beneficio es una evaluación relacionada a obtener informes de experiencias con información relevante e incluso confidencial en el desarrollo de un producto y/o servicio que se comparte entre el cliente y la organización. La transparencia se considera un componente importante ya que esconder la información tecnológica o comercial sobre servicios y/o productos puede poner en peligro el desarrollo de la confianza entre las partes interesadas (Prahalad \& Ramaswamy, 2004).

Vincular a los agro negocios con la co-creación es una temática reciente, la producción de conocimiento está más que todo relacionada a la parte productiva de los alimentos (Farinha, Bernardo, Duarte, \& Binotto, 2017).

El proceso de elaboración del producto influye en la construcción del valor de marca del producto final, desplegando estrategias que permitan desarrollar productos valiosos que sean percibidos por el cliente, lo que representa una mayor ventaja competitiva y una relación más cercana entre consumidor y el producto. Sin embargo, son pocos los estudios que apoyan el valor de marca en los productos agrícolas, por ejemplo, esto sucede con los principales productos que son exportados en Brasil (De Oliveira \& Spers, 2018).

Por otro lado, en los últimos años el avance de la tecnología de la comunicación se ha venido desarrollando de manera exponencial, dando lugar a las redes sociales, las cuales son comunidades en línea que ofrecen distintas formas de consumir contenido, compartirlo con otras personas con fines determinados, teniendo comportamientos distintos dependiendo del tipo de usuario al que va destinado el contenido (Habibi, Laroche, \& Richard, 2014; Rialti, Caliandro, Zollo, \& Ciappei, 2018).

El uso de redes sociales no solo influye en la forma como se comunican las personas sino también es un medio a través del cual muchas empresas o negocios pueden hacer llegar sus productos y servicios hacia sus consumidores finales, por ello es importante que las empresas no minimicen el gran aporte que brindan las redes sociales en términos de comercio electrónico (Alderete \& Jones, 2019).

En las últimas décadas han hecho su aparición muchas redes sociales especializadas en diferentes campos, tales como: actividades académicas, empresariales y también en los deportes. Esto ha permitido a las personas integrarse a diversos grupos sociales, eligiendo ellos el espacio donde más desean comunicarse e interactuar (Hütt, 2012).

Las redes sociales permiten que las personas y las organizaciones formen comunidades donde pueden interactuar, compartir información relevante y generar confianza, haciendo que los miembros de la red puedan entablar relaciones de colaboración mutua (Cruz \& Miranda, 2020) 
Las redes sociales ayudan a las empresas, en el sentido de tener presencia en los medios digitales, siendo un canal importante tanto para clientes como para las empresas, ya que los clientes pueden encontrar a las empresas que están buscando, y de la misma forma, la empresa está buscando justamente a esos clientes que necesita para impulsar su negocio. A su vez, las personas están inmersas en las redes sociales más importantes, lo cual es fundamental para las empresas que están buscando compradores modernos y actualizados en el uso del internet (Ipsos, 2020).

Después de todo lo señalado, el presente estudio tiene los siguientes objetivos:

- Conocer el potencial de la co-creación de valor mediante las redes sociales de los pequeños agronegocios de los agricultores rurales.

- Determinar las principales motivaciones del pequeño productor rural para realizar la co-creación de valor por medio de las redes sociales

- Determinar los principales beneficios de un producto agrario que ha pasado por un proceso de co-creación.

\section{MÉTODOS}

La investigación tiene un enfoque cualitativo, tipo básica, diseño no experimental porque se observan fenómenos en su contexto natural sin modificación de variables (Hernández, 2014). Para la recopilación de la información se realizaron entrevistas semiestructuradas.
La información recopilada fue registrada mediante grabaciones, las cuales fueron analizadas para lograr los resultados. La población de estudio estuvo conformada por individuos relacionados con el programa Agro Rural. En la Tabla 1, se detalla la cantidad y perfil de personas que formaron parte de la investigación, como informantes claves.

\section{RESULTADOS}

Después de analizar la información recopilada a partir de las entrevistas realizadas se determinó que para conocer el potencial de la co-creación de valor mediante las redes sociales de los pequeños agronegocios de los agricultores rurales, se debe tomar en cuenta cuatro aspectos relevantes, como son: determinar el potencial del productor para aplicar la co-creación de sus productos, conocer la motivación de los productores rurales, detallar la factibilidad para la co-creación mediante las redes sociales y establecer las condiciones para aplicar la co-creación en las redes sociales por parte del pequeño productor rural (Ver Figura 1).

A continuación, se describen los resultados encontrados relacionados al potencial de co-creación de valor en los pequeños agronegocios rurales. La Figura 1 muestra la representación gráfica de las categorías encontradas. La descripción de estos hallazgos se apoya en citas textuales de las entrevistas a los participantes. Se indica en cada una de ellas a quien pertenece con las iniciales de los mismos, seguida de la codificación realizada a cada entrevistado detallado en la Figura 1.

Tabla 1

Descripción de los entrevistados

\begin{tabular}{lll}
\multicolumn{1}{c}{ Codificación } & \multicolumn{1}{c}{ Informante clave } & \multicolumn{1}{c}{ Iniciales } \\
\hline E1a, E1b, E1c, E1d, E1e, E1f. & 06 productores agrarios perteneciente al programa Agro Rural & IGBH, PVR, MAQ, RT, MELQ, LER. \\
\hline E2a & 01 presidentes de asociaciones que participan del programa Agro Rural & JP \\
\hline E4a & 01 promotor del programa Agro Rural & AEM \\
E5a, E5b, E5c, E5d & 04 especialistas de Marketing digital & CR, MZ, CR, JP \\
\hline E6a, E6b & 02 especialistas en redes sociales & AM, FA \\
\hline Total & 14 personas entrevistadas & \\
\hline
\end{tabular}

Nota: Elaboración propia a partir de los datos de los participantes. 
Co-creación de valor en pequeños agronegocios a través de redes sociales
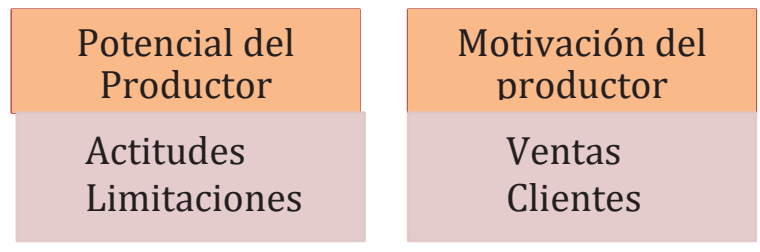

Figura 1. Potencial de co-creación de valor en los pequeños agronegocios. Elaboración propia a partir del análisis de las entrevistas.

\section{Potencial del productor.}

Los productores demuestran una actitud positiva a la co-creación por medio de las redes sociales, puesto que indican que los comentarios y reclamos de los clientes constituyen una oportunidad de mejora y además indican que conocen las redes sociales y lo utilizan de manera frecuente.

...hay comentarios que son buenos y a veces que también que nos ayudan a ver que, si hay un tema que mejorar, recibimos los comentarios sean buenos o malos, sean buenas las críticas o malas viendo el tema de poder mejorar... (LER, E1f)

En dos o tres ocasiones comentarios sobre que mi producto no es original, de porqué el frasco no está lleno en su totalidad (...) ante estos comentarios procedí a explicarles que la miel se envasa de acuerdo a lo que uno ve en las grandes tiendas de afluencia aquí en Barranca (...) siempre de la mejor manera para no perder clientes. (PVR, E1b)

$\mathrm{Si}$, son el facebook, whatsapp, tiktok (IGBH, E1a) "A mi manera, si un poco" (PVR, E1b) Facebook lo uso bastante casi todos los días (RT, E1d) Todos los días estoy conectado desde el celular (BH, E1h) Whatsapp diario y Facebook cada 3 días. (JP, E2a)

Un limitante que presentan los productores es que manejan sus redes sociales desde su perfil personal, sin diferenciarlo de su negocio, además no aprovechan de las diferentes funcionalidades y herramientas que tienen las redes sociales en beneficio del negocio.

Los productores deberían pedir ayuda a alguien que si sabe el manejo de las redes sociales para que ellos puedan alcanzar metas sobre las ventas programadas que se hayan hecho, y no es por un tema de moda sino por necesidad, siempre los productos deben mantener la calidad de los productos. (AEM, E4a)

Desde la perspectiva de los expertos, la co-creación de valor de los productos agrarios a través de redes sociales si sería posible, siendo favorable para la construcción y recordación de las marcas, para ello los productores deben desarrollar un concepto de marca de sus productos.

Es una estrategia que veo en empresas grandes como Coca Cola que ya están posicionadas, y lo único que les falta es el tema de fidelización del cliente, es una estrategia buena, pero debes saber cómo manejarla, ya que te brinda ideas como moldear tu producto final. (CR, E5c)

Definitivamente, por lo menos en la recordación de marca es un hecho, siempre y cuando desarrolle un producto que sea atractivo, es decir una plataforma que sea atractiva, que tengas muy claro a quién te diriges, eso es prioritario. (CR, E5a)

\section{Motivación del productor a co-crear en redes sociales.}

El productor demuestra motivación hacia la co-creación si esta actividad logra promover la venta de sus productos, por ejemplo, obtener mejores espacios o lugares de venta, una mayor exposición que tendrían sus productos, mayores oportunidades de venta. Otro elemento de motivación para aplicar la co-creación está relacionado con los clientes; la confianza con ellos, un mayor reconocimiento, tener una red de contactos más grande y las recomendaciones. 
El futuro de la comunicación y las ventas era el e-commerce, este futuro se ha acelerado, ahora las redes sociales cumplen un rol fundamental para todo negocio que quiere subsistir, ya que el contacto con el cliente son los medios digitales ósea las redes sociales" (JP, E5d). "Ayuda a saber que existen estas empresas pequeñas, el uso de redes sociales es gratuito y esto genera que tengas más fans de a poco. También ayuda al tema de ventas más reconocidos. (CR, E5c)

Proporción de espacios de venta, puntos de venta, acceso al cliente, mercados (MAQ, E1c).

\section{Factibilidad para la co-creación mediante las redes sociales.}

Las redes sociales conforman espacios adecuados para realizar la co-creación de cualquier producto, puesto que facilitan a los consumidores expresar sus experiencias con el producto de manera instantánea, dando la oportunidad al productor de tener acceso a estos comentarios que posteriormente servirán para aplicar mejoras de los productos.

Yo creo que para lo que fueron hechas las redes sociales, creo que una de las bases es la co-creación de valor, porque las redes sociales nos permiten tener una interacción prácticamente en tiempo real con las personas o hasta con los clientes... nos permite ese feedback directo con las personas. Anteriormente como empresa publicábamos en un periódico o revista, pero no teníamos ese feedback del consumidor. Ahorita las redes sociales hasta te permite saber cómo a tu consumidor le gustaría tener su producto y de hecho de eso se trata la co-creación, de involucrar a todos lo que forma parte de ese sistema para que entre ellos creen una mejor idea o un mejor producto y así vas añadiendo calidad a tu negocio... ( FA, E6b)

Así mismo, un producto co-creado a través de redes sociales tiene atributos relacionados con las estrategias para construir una marca, para ello los productores deben humanizar su marca aprovechando las sugerencias que los seguidores otorgan en las redes sociales.
Es importante humanizar la marca, si por ejemplo la marca tiene una historia atrás, que la cuenten, si tienen una chacra de paltos que ha pasado por una evolución familiar...esa historia tiene mucho valor, a la gente le interesa que les cuentes historia, que les cuentes cosas diferentes, que los motives, que los emociones. (CR, E5a)

...Nosotros diseñábamos imágenes para mostrar cómo era el proceso de producción de los alimentos, tanto como para las personas como para los animales...y el beneficio que le daban las redes sociales es que la gente conoció realmente cuales eran los procesos internos para tener el producto final, lo cual le daba un contenido de valor y le daba relevancia al negocio y eso ayuda a que puedas levantar tu marca, y posicionar tu marca. (FA, E6b)

Se tiene dos tipos de redes dependiendo de qué tipo de consumidor quieres llegar, están los estratos de sociedad y estilo de vida. Si quieres que te ayude a vender productos de marca de tipo estilo de vida, la ideal sería Instagram, sin embargo, si los productos son papas, esto es más promovido por facebook, y en youtube puedes hacer videos sobre tu proceso de producción. (MZ, E5b)

\section{Condiciones para la co-creación en redes sociales.}

Los expertos señalan que aquellos agronegocios interesados en desarrollar un proceso de co-creación deben cumplir ciertas condiciones, que básicamente tienen que ver con el plan estratégico, el cual pasa por conocer las debilidades y fortalezas, teniendo claro a donde se desea llegar con el negocio y qué acciones concretas están dispuestas a desarrollar los productores para lograrlo. Otra condición importante es involucrar en el negocio a personas especializadas en el manejo de las redes sociales, para asegurar que la actividad de co-creación se realice de manera profesional.

Para crear valor, primero debes tener en claro cuál es tu visión y misión, tus valores, porque la marca ahora no es solo un nombre o logotipo, es un personaje con 
valor...luego debes comenzar a interactuar con esos usuarios. (JP, E5d)

Se necesita focalizar que tipo de negocio es. Lo primero es definir o separar cada tipo de producto..., iniciar con un plan de comunicación donde se identifique quien es la persona que te va a dar valor. (MZ, E5b)

Conocer a tu producto, saber qué es lo que hace que tu producto sobresalga, tener un contacto con tus clientes, siempre y cuando sabiendo si ellos quieren comprar tu producto. (CR, E5c)

Debe haber personas especiales o área especializada que se encargue del manejo de las redes sociales. Estudiar toda la información que ha recibido, analizarla, ejecutarla. (AM, E6a)

\section{DISCUSIÓN}

Los hallazgos de esta investigación brindan un panorama sobre el potencial de la co-creación de valor en redes sociales de los pequeños agronegocios de los productores rurales, demostrando que estos últimos tienen una actitud positiva hacia la co-creación, puesto que están dispuestos a escuchar a sus clientes para conocer las opiniones referentes a sus productos, e incluso escuchar sus críticas, pues ellos lo consideran como una oportunidad para mejorar. Este aspecto está acorde con el propósito de la co-creación, como lo señalan Jiménez-Barreto \& Campo-Martínez (2018) cuando afirman que la co-creación tiene su esencia en la satisfacción del consumidor. En este mismo orden de ideas, Bettiga, Lamberti, \& Noci (2018) recalcan la importancia de hacer participar a los consumidores en el desarrollo de productos, ya sea en el diseño, el precio o la promoción de los mismos, de esa manera generar valor para el negocio.

A pesar, que los productores tienen buena disposición para participar en procesos de co-creación, son evidentes las limitaciones que ellos tienen para realizarlo por medio de las redes sociales, puesto que necesitan desarrollar sus habilidades en el uso de las herramientas digitales. Por lo tanto, los productores tienen el potencial de crear valor de sus productos mediante estas redes, siempre y cuando encuentren con las oportunidades, conocimiento y ayuda especializada en estos temas.

Los productores sienten una motivación por incrementar sus ventas y el número de sus clientes, lo que significa que disponen de producción para poder ofrecerla al mercado y buscan la oportunidad para poder hacerlo. Las redes sociales significan una oportunidad para lograr más ventas, tal como señala Cruz y Miranda (2020), estas redes son comunidades que permiten interactuar con las personas y recoger las opiniones que ayuden en la mejora de los productos, ya sea en empaque, presentación, calidad entre otros aspectos.

Un aspecto muy importante de la co-creación de los productos mediante las redes sociales es el desarrollo de marca. puesto que la innovación desarrollada, de la mano de los seguidores, brinda los argumentos suficientes para el contenido de las publicaciones en redes sociales, de manera transparente y participativa. Esto va alineado a lo señalado por Donato, Farina, Donaire y Dos Santos (2017) quienes afirman que la transparencia es un componente clave al momento de brindar información en redes sociales, la que puede ser verídica en cualquier momento. Todas estas actividades brindan la oportunidad de desarrollar productos valiosos (De Oliveira \& Spers, 2018), diseñados de acuerdo a las necesidades y expectativas de los consumidores.

Es importante recalcar que para lograr la co-creación de los pequeños agronegocios en redes sociales es esencial realizar un plan estratégico, partiendo primeramente por determinar las debilidades y fortalezas de los productos y posteriormente proyectar objetivos concretos a largo y mediano plazo, estableciendo la misión y visión del negocio.

\section{CONCLUSIONES}

La humanidad está trabajando bajo una agenda que se pretende alcanzar al 2030, entre los objetivos que se intenta cumplir están: poner fin a la pobreza, hambre cero, lograr el trabajo decente y desarrollo económico, entre otros.

La agricultura familiar emplea a un gran sector de la población en condiciones de pobreza. Sin embargo, estos pequeños productores 
contribuyen con las necesidades alimentarias en distintas partes del mundo, por ello se hace urgente garantizar la sostenibilidad de la cadena de valor que puede beneficiar a millones de hogares pobres en los países en desarrollo.

Al igual que en diferentes partes del mundo, el gobierno peruano busca promover el desarrollo de la agricultura familiar y por ello se tienen normas, leyes y reglamentos que la promueven, así mismo se cuenta con el Programa de desarrollo Productivo Agrario Rural, Agro Rural, que busca, entre otras acciones, desarrollar actividades que logren insertar al pequeño productor al mercado.

Para lograr que los negocios se inserten al mercado, crezcan y se desarrollen, existen diversas estrategias, una de ellas es la co-creación, que a pesar que no es un término nuevo, permite desarrollar diversas acciones de innovación que las empresas pueden desarrollar de la mano con sus grupos de interés, es por ello que en los últimos tiempos está siendo de interés para empresarios, académico e investigaciones.

Gracias al avance de las tecnologías, las redes sociales se han constituido como un medio que permite interactuar entre empresas y consumidores, lo que constituye una oportunidad para desarrollar acciones de co-creación de los productos agrarios.

Los productores rurales presentan una buena disposición a realizar acciones de co-creación de valor por medio de las redes sociales, pero necesitan adquirir conocimientos sobre tecnología.

Realizar mejoras de los productos agrarios a partir de la co-creación con los clientes genera oportunidades para la creación y desarrollo de marcas, las cuales son un activo muy importante que los productores rurales podrían aprovechar.

Ayudar al desarrollo del sector de la agricultura familiar es contribuir al logro de los objetivos de desarrollo sostenible, puesto que se genera trabajo, mejora su ingreso y se lucha contra el hambre y la desnutrición, así como se genera el trabajo digno y la igualdad de oportunidades, es por ello que se debe realizar más investigaciones relacionadas al tema para seguir apoyando a este sector.

\section{REFERENCIAS BIBLIOGRÁFICAS}

Alderete, M. V., \& Jones, C. (2019). ¿Hacia el social commerce? El valor de las redes sociales en la MiPyME de Córdoba, Argentina. Entramado, 15(1), 48-60. https://doi.org/10.18041/19003803/entramado.1.5149

Bettiga, D., Lamberti, L., \& Noci, G. (2018). Investigating social motivations, opportunity and ability to participate in communities of virtual co-creation. International Journal of Consumer Studies, 42(1), 155-163. https://doi. org/10.1111/ijcs.12409

Castro, H. L., Goicochea, C. U., \& Flores, M. F. (2018). El Sistema De Agronegocios En El Peru: De La Agricultura Familiar Al Negocio Agroalimentario. Revista Mexicana de Agronegocios, 43(2018), 1-16. https://www.redalyc.org/jatsRepo/141/14158242001/html/index.html

Comisión Económica para América Latina y el Caribe, La Organización de las Naciones Unidas para la Alimentación y la Agricultura y el Instituto Interamericano de Cooperación para la Agricultura (2019) Perspectivas de la agricultura y del desarrollo rural en las Américas: una mirada hacia América Latina y el Caribe 2019-2020. San José, Costa Rica. Retrieved from http://repositorio.iica.int/bitstream/handle/11324/8214/ BVE19040295e.pdf; jsessionid=CCE1AE90583C7B1642AEC46BEBC478B6? sequence $=1$

Ley N. ${ }^{\circ}$ 997. Ley de organización y funciones del Ministerio de Agricultura. Congreso de la Republica del Perú. Diario oficial El Peruano, 13 de marzo 2008. Recuperado de http://www.ana. gob.pe/sites/default/files/normatividad/files/ dleg_997_0_0.pdf

Convención Nacional del Agro Peruano (2018). Ley de Promoción y Desarrollo de la Agricultura Familiar - LEY - N 30355. Diario El Peruano.

Cruz, I., \& Miranda, A. (2020). Importancia de la inclusión de las estrategias con redes sociales digitales en restaurantes de la zona gastronómica de la ciudad de Tijuana. El Periplo Sustentable, (38), 74 - 112.https://doi.org/DOI:10.36677/elperiplo.v0i38.9947.

De Oliveira, R. O., \& Spers, E. E. (2018). Brand equity in agribusiness: Brazilian consumer perceptions of pork products. RAE Revista de Administracao de Empresas, 58(4), 365-379. https:// doi.org/10.1590/S0034-759020180403

Donato, H., Farina, M., Donaire, D., \& Dos Santos, I. (2017). 2017 • ISSN 1678-6971 (electronic version) • Revista de Administração Mackenzie-RAM (Mackenzie Management Review). 
Mackenzie Management Review), 18(5), 63-91. https://doi.org/10.1590/1678-69712017/administracao.v18n5p63-91

Organización de las Naciones Unidas para la Alimentación y la Agricultura. (2018). Agronegocios y cadenas de valor alimentarias Sostenibles |. Recuperado de http://www.fao.org/policy-support/policy-themes/sustainable-agribusiness-food-value-chains/es/

Farinha, J., Bernardo, L., Duarte, N., \& Binotto, E. (2017). Agribusiness cocreation: A systematic review. Espacios, 38(29), 29. Recuperado de https://www.scopus.com/inward/record.uri?ei$\mathrm{d}=2$-s2.0-85021363825\&partnerID $=40 \& \mathrm{~m}$ $\mathrm{d} 5=0 \mathrm{f} 02 \mathrm{db} 44 \mathrm{c} 1 \mathrm{fc} 86443 \mathrm{c} 0 \mathrm{a} 440 \mathrm{e} 25956766$

Flores Calle, A. de los M. (2014). Desempeño del subsistema de agronegocios de palta Hass peruana.

Habibi, M. R., Laroche, M., \& Richard, M. O. (2014). The roles of brand community and community engagement in building brand trust on social media. Computers in Human Behavior, 37, $152-$ 161. https://doi.org/10.1016/j.chb.2014.04.016

Handayati, Y., Simatupang, T. M., \& Perdana, T. (2015). Value Co-creation in Agri-chains Network: An Agent-Based Simulation. Procedia Manufacturing, 4, 419-428. https://doi.org/10.1016/j.promfg.2015.11.058

Hernández, R., Fernández, C., y Baptista, M. (2014). Metodología de la investigación. México: McGrawHill Education. Recuperado de https:// periodicooficial.jalisco.gob.mx/sites/periodicooficial.jalisco.gob.mx/files/metodologia_de_ la_investigacion_-_roberto_hernandez_sampieri.pdf

Hütt, H. (2012). Las Redes sociales: Una nueva herramienta de difusión. Reflexiones, 91(2), 121-128. https://www.redalyc.org/ pdf/729/72923962008.pdf

Instituto Interamericano de Cooperación para la Agricultura IICA. (2010). Desarrollo de los agronegocios y la agroindustria rural en América Latina y el Caribe. IICA. San José, Costa Rica. https://doi.org/10.1049/PBHE001E_ch22
Ipsos. (2020). Redes sociales en el Perú Urbano. Recuperado de https://www.ipsos.com/es-pe/ redes-sociales-en-el-peru-urbano

Jiménez-Barreto, J., \& Campo-Martínez, S. (2018). Destination website quality, users' attitudes and the willingness to participate in online co-creation experiences. European Journal of Management and Business Economics, 27(1), 26-41. https://doi.org/10.1108/EJMBE-11-2017-0048

Loureiro, S., Romero, J., \& Bilro, R. (2019). Stakeholder engagement in co-creation processes for innovation: A systematic literature review and case stud. Journal of Business Research. https:// doi.org/10.1016/j.jbusres.2019.09.038

Martini, A., Massa, S., \& Testa, S. (2012). The Role of Social Software for Customer Co-Creation: Does it Change the Practice for Innovation? International Journal of Engineering Business Management, 4(40), 1-9. https://doi. org/10.5772/54750

Naciones Unidas. (2018). La Agenda 2030 y los Objetivos de Desarrollo Sostenible: una oportunidad para América Latina y el Caribe. Objetivos, metas e indicadores mundiales. Publicación de las Naciones Unidas. Santiago. Recuperado de https://repositorio.cepal.org/bitstream/handle/11362/40155/24/S1801141_es.pdf

Prahalad, C. K., \& Ramaswamy, V. (2004). Co-creation experiences: The next practice in value creation. Journal of Interactive Marketing, 18(3), 5-14. https://doi.org/10.1002/dir.20015

Rialti, R., Caliandro, A., Zollo, L., \& Ciappei, C. (2018). Co-creation experiences in social media brand communities: Analyzing the main types of co-created experiences. Spanish Journal of Marketing - ESIC, 22(2), 122-141. https://doi. org/10.1108/SJME-03-2018-0011 
